\title{
The Bone Conduction Microphone Parameter Measurement Architecture and Its Speech Recognization Performance Analysis
}

\author{
Hang-Hong Kuo ${ }^{1, a^{*}}$, Ying-Yu Yu ${ }^{1, a}$ and Jun-Juh $\mathrm{Yan}^{2, \mathrm{~b}}$ \\ ${ }^{1}$ 14F, No. 1-85, Zhonghua Rd., Yongkang Dist., Tainan City 71084, Taiwan(R.O.C.) \\ ${ }^{2}$ No.59, Hengshan Rd., Yanchao Dist., Kaohsiung City 82445, Taiwan(R.O.C.) \\ acliff kuo@neovictory.com, ${ }^{\mathrm{b}}$ davis yu@neovictory.com, ${ }^{\mathrm{c}}$ ĳyan@stu.edu.tw \\ * corresponding author
}

Keywords: Bone conduction microphone. Parameter measurement architecture. Anti-noise. Speech recognition.

Abstract. Bone conduction microphone (BCM) can robustly resist noisy background. In this paper, an industrial parameter measurement architecture has been proposed to measure the main parameters of BCM. For comparing the robustness of BCM, a synchronized record platform has been developed. To observe the speech identification of BCM, a smart phone-based software has been designed and verified with Google ASR.

\section{Introduction}

From 1990s, as the communication between people becomes more important in human life, the correlated products have been massively manufactured, such as the walkie-talkie, mobile phone, PDA (personal digital assistants), tablet PC, smart phone, Bluetooth handset, WiFi Audio/Video transmitter, etc. The communication technology also has been changed from analog to digital field, includes the Internet in recent decade. As the results, these extend applications provide the long distance Audio/Video conference, speech recognition and remote control, personal verification, etc. The most important element of these applications is the high identification speech signal.

For obtaining the high identification speech and precise speech recognition, the clear speech should be collected. The acoustic speech researches have been popular studied in last decade, such as AEC (acoustic echo canceler, or audio echo cancellation) [1], ANC (audio noise cancellation) [2], speech detection [3]. The general knowledge of speech/audio collector is the air conduction microphone (ACM). It brings several advantages, such as high quality, wide range of frequency response, and convenient to use. However, it also exists a main inferiority: low anti-noise ability of collecting process which will limits the operating field of ACM. Many pioneer researchers had explored enhanced methods to improve the resolving of collecting/collected speech/audio with single, dual, or multiple microphones [1]. In these studies, the extra processor unit or ACM element is requisite to assist the main ACM to obtain the clear speech/audio actively.

In this paper, we adopt another microphone element, called bone conduction microphone (BCM), to pick up the speech of speaker. The picked up speech which passed through the vibration of human skull has different conducting path to ACM. Therefore, BCM is not sensitivity to external interference, such as background noise. Moreover, the quality of collected speech from BCM will be varied while the position of BCM changed [4]. Although the BCM is robust to noise effect, several studies pointed out its infirm to high-frequency of speech which caused by the conduction path loss [5]. For these reasons, the study of frequency parameter adjustment and improvement becomes important.

This paper presents a parameter measurement architecture of BCM and its real-world measurement result of NeoVictory technology Co., Ltd. BCM product. Moreover, an ACM and BCM combination platform has been set up to record and compare their anti-noise ability. And the speech recognition of ACM and BCM are also studied. 


\section{Bone conduction microphone and its parameter measurement architecture}

This paper presents a parameter measurement architecture of BCM and its real-world measurement result of NeoVictory technology Co., Ltd. BCM product. Moreover, an ACM and BCM combination platform has been set up to record and compare their anti-noise ability. And the speech recognition of ACM and BCM are also studied.

\section{A. The Structure of Bone Conduction Microphone}

The speech collect of BCM is based on mechanism to electronic conversion. The BCM usually constitutes by a body, a piezoelectric ceramic plate as positive pole, and a metal plate as negative pole. Fig. 1. and Fig. 2. show the schematic and BCM product of NeoVictory Co..
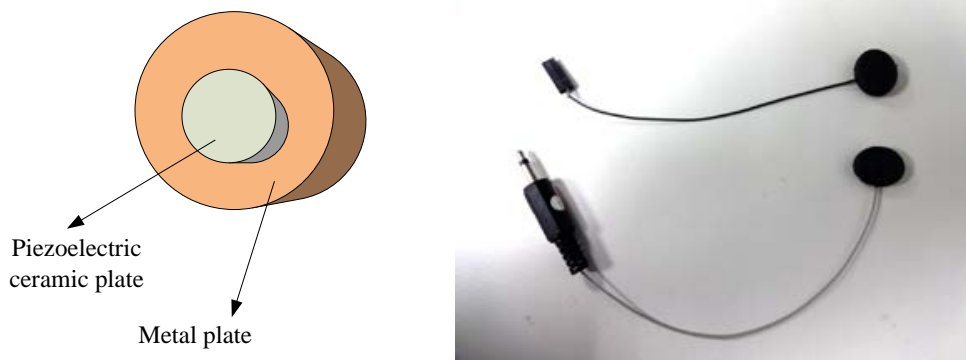

Fig. 1. Schematic of BCM. Fig. 2. BCM product of NeoVictory Tech. Co.

\section{B. Parameter Measurement Architecture of Bone Conduction Microphone}

The BCM is a vibration to electricity energy convert. Its related parameters are impedance / capacitance / sensitivity / spectrum. These parameters will be measured with their corresponding instrument.

The impedance and capacitance can be measured by Ohmmeter and capacitance meter, respectively. The sensitivity and spectrum should be measured by the proposed architecture as below. In Fig. 3, beside the BCM, the hardware of architecture should include power supply, vibration driver, loading, buffer, standard vibrator, vibration sensor and sensor amplifier. The main functions are briefly described as follows:

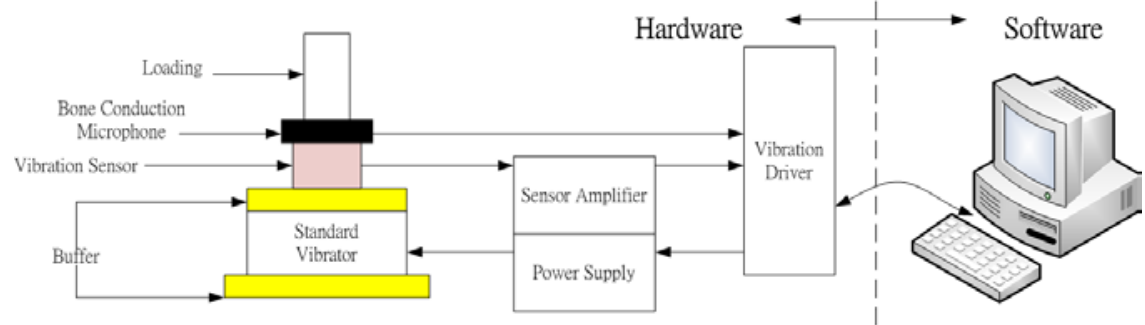

Fig. 3. Parameter measurement architecture of BCM.

- Power supply: provide the power source to standard vibrator.

- Vibration driver: receive the command from software and drive the standard vibrator to generate the organized sinusoid tone to vibration sensor or BCM.

- Loading: give a constant pressure force on the BCM.

- Buffer: digest the vibration from ground by using $5 \mathrm{~cm}$ sponge.

- Standard vibrator: generate the specific or organized sinusoid tone.

- Vibration sensor: regulate and amend the vibration between sensor amplifier and vibration driver.

- Sensor amplifier: adjust the multiple coefficients between vibration sensor and vibration driver.

The software of architecture is the CLIOwin platform on personal computer. For starting the parameter measurement of BCM, the normalization of machine should be completed first.

After normalization, the parameter measurement should start as below steps:

(1) Switch CLIOwin on software platform to spectrum measurement.

(2) Send the organized sinusoid tone, from $10 \mathrm{~Hz}$ to $20 \mathrm{kHz}$.

(3) Measure and store its spectrum. 


\section{Test and Record Platform of Air Conduction Microphone and Bone Conduction Microphone}

\section{A. Proposed Test and Record Platform of ACM and BCM}

As the general knowledge, the ACM has high sensitivity of speech/audio, but low anti-noise ability of noisy background. Oppositely, the BCM has good noise robust ability of background influence, but high frequency components are attenuated critically which caused by path loss. This sector purposes a combination platform for testing and recording ACM and BCM collected speech which un-interfered / interfered by background noise. The schematic of test and record platform is shown in Fig. 4. And Fig. 5 shows the implemented platform.

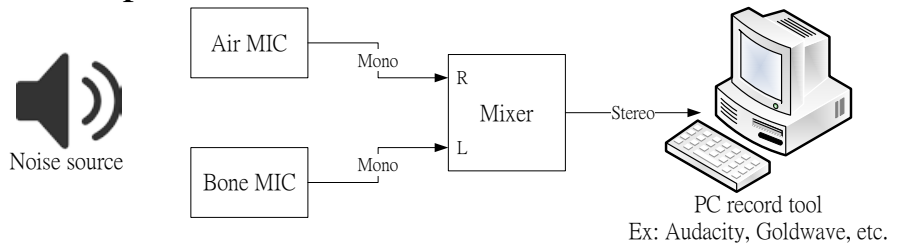

\section{B. Speech Recognition Platform for BCM}

Fig. 4. The schematic of test and record platform for ACM and BCM.

Based on the proposed platform in Fig. 5., the recorded speech is also used to verify its speech identification with ASR, Android Speech Recognition, on Android smart phone. The realized hard platform is shown as Fig. 6. Its software recognition screen is captured as shown in Fig. 7.

The WER, word error rate, is used to verify the difference between target word and result.

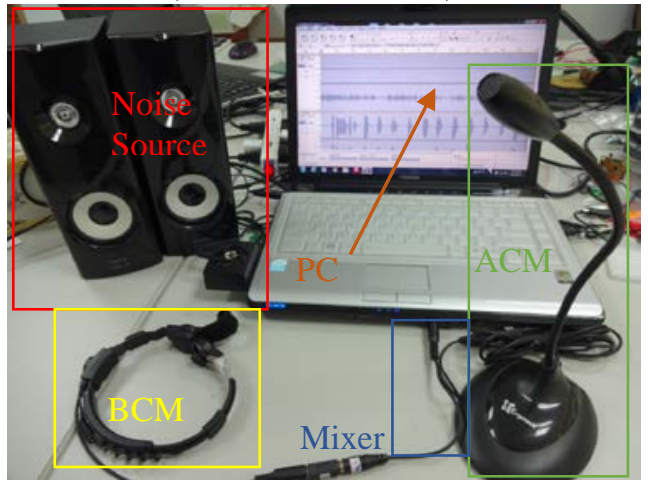

Fig. 5. The implementation record platform.
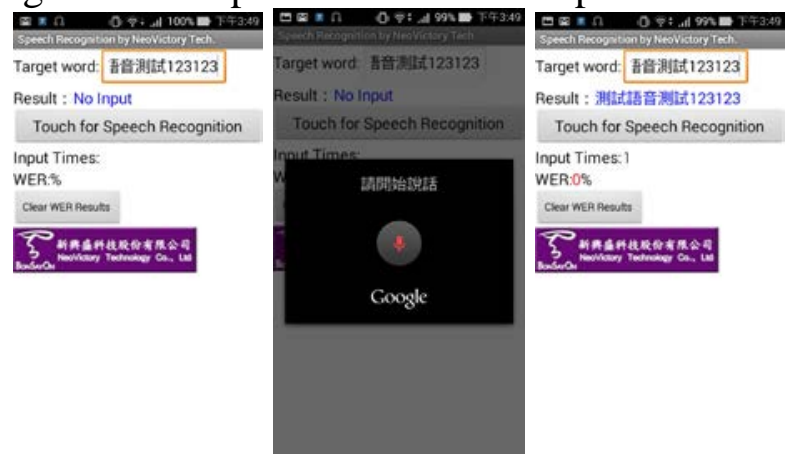

(a) Enter target word (b) record speech (c) result Fig. 7. The speech recognition screenshot on smart phone.

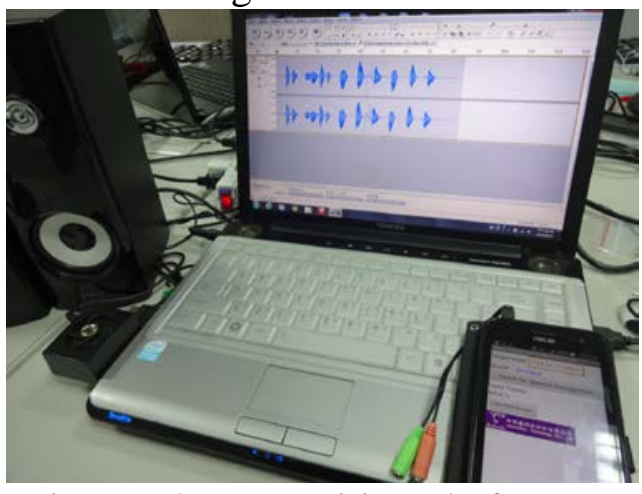

Fig. 6. The recognition platform.

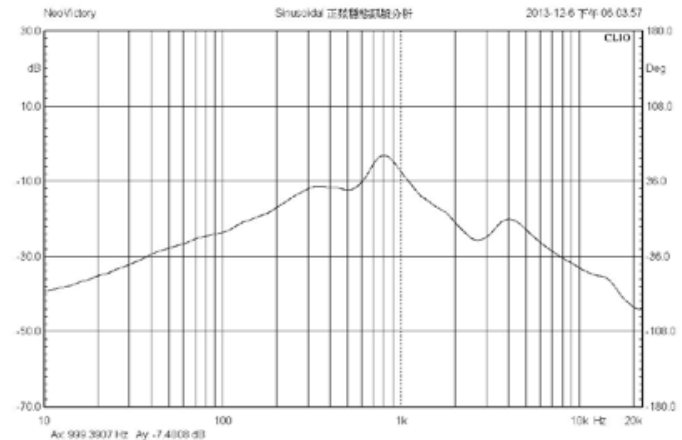

Fig. 8. Frequency response of NeoVictory BCM.

\section{Experimental Results}

As the illustrations in section II and III, the experiments are separated to three parts. The first is the parameter measurement platform, TABLE 1 shows the measured parameters of NeoVictory BCM product, and its frequency response is shown as Fig. 8.

The second part is the record results compared in three environments: quiet office room, nearby noisy rail, noisy wind. TABLE II shows the volume decibel of these environments. Figs. $9 \sim 11$ show the recorded speech waveforms. 
Table I. Parameters of NeoVictory BCM

\begin{tabular}{|l|c|}
\hline \multicolumn{1}{|c|}{ Parameter } & Value \\
\hline Output impedance & $10 \mathrm{~K} \Omega$ \\
\hline Capacitance & $76 \mathrm{pF}$ \\
\hline Sensitivity & $-10 \mathrm{~dB}$ \\
\hline
\end{tabular}

Table II. Decibel of testing environments

\begin{tabular}{|l|c|}
\hline \multicolumn{1}{|c|}{ Parameter } & Value \\
\hline Quiet office room & $40 \mathrm{~dB}$ \\
\hline Nearby noisy rail & $90 \mathrm{~dB}$ \\
\hline Noisy wind & $80 \mathrm{~dB}$ \\
\hline
\end{tabular}

In Fig.9, the top two rows are the records of BCM and ACM nearby the rail, separately, without speaking; the below two rows recorded user speaking nearby the rail, separately. From the below two rows, the noise interference picked up the speech seriously on ACM, but interference on BCM is slight. This also happens in Fig. 10. From Figs.9 and 10, the anti-noise ability of BCM is obviously better than ACM.

The third part is the recognition results of BCM. In this paper, the different language has been tested and verified. The results have been shown in TABLE III. The recognition WER of Chinese is 45\% and WER of English is 30\%, the Chinese word has worse WER than English in 30 times record. However, the larger tests might change this situation.

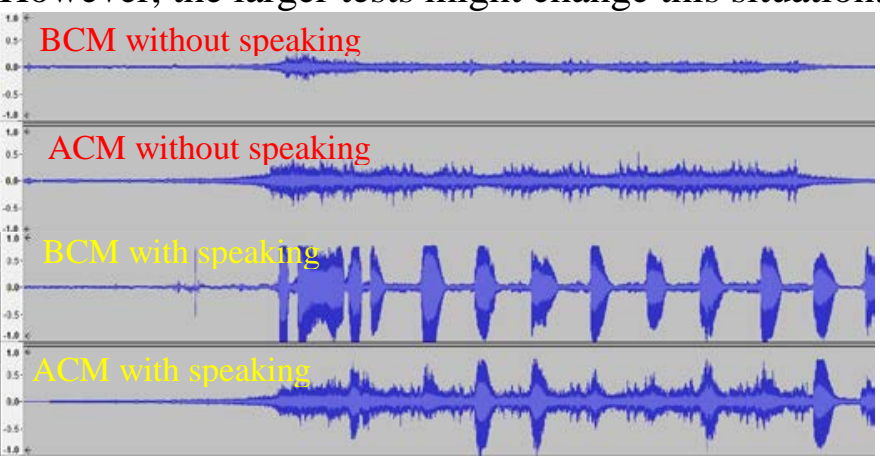

Fig. 10. The record of nearby noisy rail.

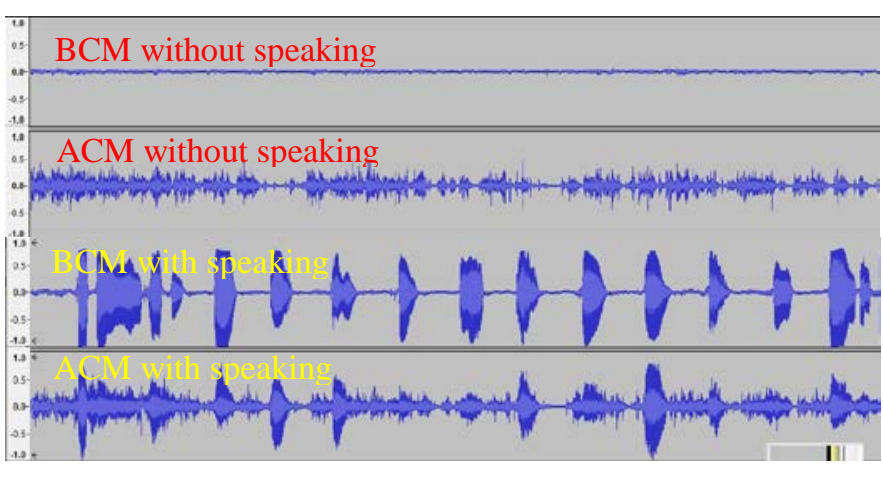

Fig. 11. The record of noisy wind.

\section{Conclusions}

In this paper, a parameter measurement architecture for BCM has been proposed. This platform can be used to measure the main parameters: impedance / capacitance / sensitivity / spectrum of BCM. Moreover, a synchronized speech/audio record platform is developed and used to record the picked up speech of ACM and BCM. Based on this platform, the noise interference to ACM and BCM is also conferred. At the end, the speech recognition software is implemented to complete the WER verification of BCM.

\section{References}

[1] P.C. Loizou, Speech Enhancement: Theory and Practice, CRC Press, 1ed., June 2007.

[2] R. Singh Rana, L. Zhang, B. Tang, and G.H. Krishna, "An enhanced method and behavioral model for noise cancellation in audio devices,” 2014 IEEE International Workshop on Biomedical Circuits and Systems, pp. S2/6 - 11-14, December 2004.

[3] B.V. Yadong, “ A noise robust speech activity detection algorithm,” Proceedings of 2004 international symposium on intelligent multimedia, video and speech processing, pp. 322-325, October2004.

[4] P. Tran, T. Letowski, and M. McBride, "Bone conduction microphone: Head sensitivity mapping for speech intelligibility and sound quality,” International conference on audio, language and image processing, pp. 107-111, July 2008.

[5] T. Shimamura and T. Tamiya, “A reconstruction filter for bone-conducted speech,” proc. Of the midwest symposium on circuits and systems, vol. 2, pp. 1847-1850, August 2005. 